\title{
Extremely short relativistic-electron-bunch generation in the laser wakefield via novel bunch injection scheme
}

\author{
A. G. Khachatryan, F. A. van Goor, and K.-J. Boller \\ Department of Applied Science, University of Twente, P.O. Box 217, 7500 AE Enschede, The Netherlands
}

A. J.W. Reitsma and D. A. Jaroszynski

Department of Physics, University of Strathclyde, 107 Rottenrow, Glasgow G4 ONG, United Kingdom

(Received 19 July 2004; published 6 December 2004)

\begin{abstract}
Recently a new electron-bunch injection scheme for the laser wakefield accelerator has been proposed [JETP Lett. 74, 371 (2001); Phys. Rev. E 65, 046504 (2002)]. In this scheme, a low energy electron bunch, sent in a plasma channel just before a high-intensity laser pulse, is trapped in the laser wakefield, considerably compressed and accelerated to an ultrarelativistic energy. In this paper we show the possibility of the generation of an extremely short (on the order of $1 \mu \mathrm{m}$ long or a few femtoseconds in duration) relativistic-electron-bunch by this mechanism. The initial electron bunch, which can be generated, for example, by a laser-driven photocathode rf gun, should have an energy of a few hundred $\mathrm{keVs}$ to a few MeVs, a duration in the picosecond range or less and a relatively low concentration. The trapping conditions and parameters of an accelerated bunch are investigated. The laser pulse dynamics as well as a possible experimental setup for the demonstration of the injection scheme are also considered.
\end{abstract}

DOI: $10.1103 /$ PhysRevSTAB.7.121301

PACS numbers: 52.38. Kd, 52.35.Mw, 41.85.Ar

\section{INTRODUCTION}

Ultrashort electron bunches with lengths on the order of a hundred microns have been produced using photocathode radio-frequency (rf) guns [1], thermionic rf guns [2], or with a magnetic bunch compressor [3]. However, many applications, such as laser wakefield acceleration, inverse free electron laser (FEL), x-ray FEL, ultrafast electron diffraction, and other applications, require much shorter electron bunches with a length on the order of $1 \mu \mathrm{m}$, which is 2 orders of magnitude less than achieved so far. With femtosecond electron bunches one can also generate femtosecond $\mathrm{x}$-ray pulses by $90^{\circ}$ Thomson scattering [4] for investigation of ultrafast phenomena. However, the generation of such an extremely short electron bunch is a difficult technical problem, which is not solved yet and new approaches to which are required.

In the laser wakefield accelerator (LWFA) a short intense laser pulse, with a duration on the order of a plasma wave period, generates an unusually strong plasma wake wave, usually called a laser wakefield [5]. This strong wakefield can be used for acceleration and/or focusing of electron bunches. Accelerating gradients as high as a few tens of $\mathrm{GV} / \mathrm{m}$ have been measured in experiments (see, e.g., Ref. [6]), that is 3 orders of magnitude higher than those achieved in conventional radio frequency linear accelerators. This makes LWFAs very attractive for electron acceleration. A plasma channel with a length of a few centimeters and a plasma density profile with a minimum on the axis are required for LWFA to guide the laser pulse; otherwise the laser-plasma interaction distance is restricted by Rayleigh diffraction length, which is typically on the order of a millimeter.
The wavelength and transverse extent of the wakefield are determined by the plasma density and the laser pulse spot size, and are typically on the order of a few tens of microns. Because the accelerating and focusing components of the wakefield are shifted in phase, only a portion of the plasma wave is available for electron acceleration. For a linear wakefield in a uniform plasma, the overlap of accelerating and focusing parts is only a quarter of the plasma wavelength. For nonlinear wakefields in uniform plasma and for linear and nonlinear wakefields in a plasma channel, the overlap is still less than half the plasma wavelength. So, the appropriate accelerating region in the laser wakefield is quite small and the field in this region changes from zero to its maximum value. Therefore, if the accelerating electron bunch size is comparable to the size of the accelerating region, the energy gain for the bunch electrons will vary over a very broad range resulting in $100 \%$ energy spread in the accelerated bunch. This is not appropriate for many applications. To avoid the large energy spread in the accelerated bunch, one needs to inject an electron bunch with a length much less than the plasma wavelength and a width essentially less than the laser pulse diameter. As mentioned above, such extremely short bunches are not available yet. Difficult technical problems with injection of a small size bunch into the laser wakefield are also the required synchronization of a femtosecond scale and the precise transverse positioning of the bunch in the wakefield. So, the electron injection is the hardest issue in the laser wakefied acceleration. There are ideas on internal electron bunch injection in LWFA, when some plasma electrons are trapped in the wakefield by the wave- 
breaking mechanism [7-9] or by additional laser pulses [10-13].

Recently a promising approach with an external electron bunch injection in LWFA has been proposed [14,15]. This LWFA scheme does not need a bunch with a duration of a few femtoseconds before acceleration, but employs the trapping and compression of a low-energy relatively long $e$-bunch injected in a plasma channel just before the laser pulse.

We show in this paper that this injection scheme allows the generation of a few femtosecond duration relativistic electron bunch with an energy up to several GeVs by the laser wakefield. The trapping conditions, parameters of the trapped and accelerated $e$-bunch, as well as the laser pulse dynamics in the plasma channel are considered. A possible experimental setup for the demonstration of this injection scheme is discussed.

\section{THE NOVEL INJECTION METHOD}

\section{A. Description of the idea and basic equations}

The main idea of the novel bunch injection method $[14,15]$ is as follows: a low-energy, relatively long and rare electron bunch (compared to the requirements for an $e$-bunch accelerating in the wakefield) is copropagating with a laser pulse and is injected in a plasma just before the pulse (see Fig. 1). Because the initial bunch velocity $\nu_{0}=c\left(1-\gamma_{0}^{-2}\right)^{1 / 2}$ (where $\gamma_{0}$ is the relativistic factor and $c$ is the speed of light in vacuum) is less than the group velocity of the laser pulse $\nu_{g}$, the bunch will be overtaken by this pulse. As it was shown earlier [14,15], the electron bunch propagates through the region occupied by the laser pulse and can be trapped, compressed, and accelerated in the laser wakefield. This approach was initially proposed

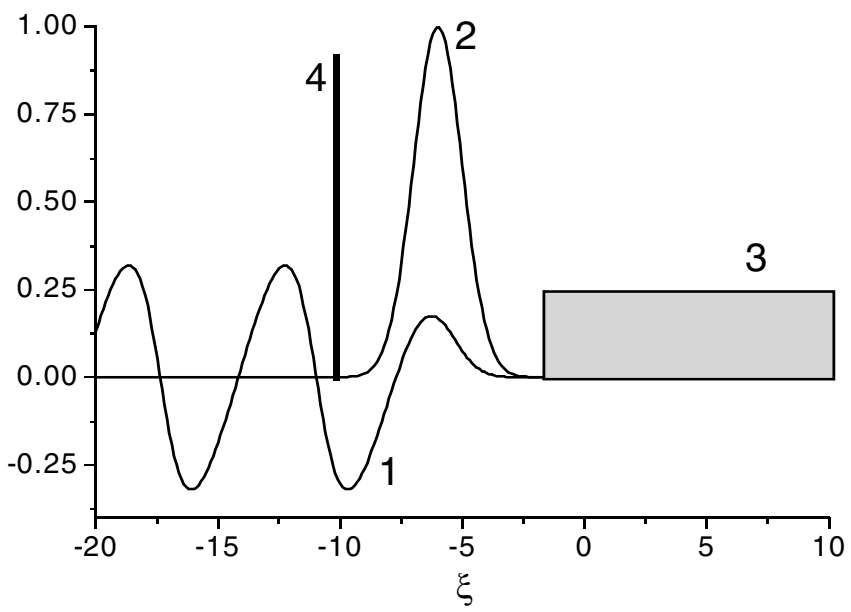

FIG. 1. The novel injection method. The normalized longitudinal (accelerating) component of the laser wakefield $E_{z}$ (curve 1), and the laser pulse intensity profile (curve 2). Areas 3 and 4 schematically show the initial and trapped electron bunches, correspondingly. in a one-dimensional setting in Ref. [14] and then generalized for the channel-guided LWFA in Refs. [15,16].

A laser pulse propagating in a plasma can be described by the normalized electric field amplitude $a(\xi, r)=$ $E_{L} /\left(m_{e} c \omega_{L} / e\right)$, where $e$ and $m_{e}$ are the absolute electron charge and mass, $\omega_{L}$ is the laser frequency, $\xi=z-\tau$. The spatial coordinates $z$ and $r$ are normalized to $1 / k_{p}$ and the time $\tau$ is normalized to $1 / \omega_{p}$, where $k_{p}=\omega_{p} / \nu_{g}$ and $\omega_{p}=\left(4 \pi n_{p} e^{2} / m_{e}\right)^{1 / 2}$ are the plasma wave number and frequency correspondingly, $n_{p}$ is the plasma electron concentration. Nonlinear axially symmetrical laser wakefields excited in a plasma channel are described by the following system of equations [17]:

$$
\begin{aligned}
\beta_{g} \frac{\partial\left(p_{e z}-\gamma_{e}\right)}{\partial \xi}-\beta_{g}^{2} E_{z} & =0, \\
\beta_{g} \frac{\partial p_{e r}}{\partial \xi}-\frac{\partial \gamma_{e}}{\partial r}-\beta_{g}^{2} E_{r} & =0, \\
-\frac{\partial\left(H_{\theta}-\beta_{g} E_{r}\right)}{\partial \xi}+\beta_{e r} N_{e} & =0, \\
\nabla_{\perp} H_{\theta}+\beta_{g} \frac{\partial E_{z}}{\partial \xi}+\beta_{e z} N_{e} & =0, \\
\frac{\partial\left(\beta_{g} H_{\theta}-E_{r}\right)}{\partial \xi}+\frac{\partial E_{z}}{\partial r} & =0, \\
\nabla_{\perp} E_{r}+\frac{\partial E_{z}}{\partial \xi}+N_{e}-N_{p} & =0,
\end{aligned}
$$

where $E_{z, r}$ and $H_{\theta}$ are the longitudinal and radial components of the electric field and the azimuthal component of the magnetic field normalized to the on-axis wavebreaking field $E_{W B}=m_{e} \omega_{p}(r=0) \nu_{g} / e, p_{e z, e r}$ are the normalized components of the plasma electron momentum, $\gamma_{e}=\left(1+p_{z}^{2}+p_{r}^{2}+a^{2} / 2\right)^{1 / 2}$ is the relativistic factor, $\beta_{e z, e r}=p_{e z, e r} / \gamma_{e}, N_{e}=n_{e}(\xi, r) / n_{p}(0)$ is the normalized density of the plasma electrons, $n_{p}(r)$ is the unperturbed plasma density in the channel, $N_{p}=$ $n_{p}(r) / n_{p}(0), \nabla_{\perp}=\partial / \partial r+1 / r$. The normalized force acting on the relativistic electrons in the wakefield is $\mathbf{F}=$ $\left(-E_{z},-\left(E_{r}-\beta_{g} H_{\theta}\right), 0\right)$. It follows from expression (1e) that $\boldsymbol{\nabla} \times \mathbf{F}=0$, such that $\mathbf{F}$ can be written as $\mathbf{F} \propto \boldsymbol{\nabla} \phi$, where

$$
\phi=-\beta_{g}^{2} \int_{\xi}^{+\infty} E_{z} d \xi
$$

is the normalized potential. The laser pulse is assumed to have a Gaussian profile:

$$
a(\xi, r)=a_{0} \exp \left[-\left(\xi-\xi_{c}\right)^{2} / \sigma_{z}^{2}-r^{2} / \sigma_{r}^{2}\right],
$$

$\xi_{c}$ corresponds to the center of the pulse. The plasma wake wave generated behind the laser pulse has a maximum amplitude when $\sigma_{z}=2$ in the dimensionless units. The laser wakefield amplitude $E_{z \text {,max }}$ is proportional to $a_{0}^{2} /\left(1+a_{0}^{2} / 2\right)^{1 / 2}[5]$. The laser pulse is assumed to propa- 
gate in a preformed parabolic plasma channel with the following unperturbed electron density profile [18]:

$$
N_{p}=1+\Delta \frac{r^{2}}{r_{c h}^{2}}
$$

where $r_{c h}$ is the channel radius and $\Delta=\left(2 / \sigma_{r} \beta_{g}\right)^{2}$. The matched laser pulse radius corresponds to $\sigma_{r}=r_{c h}$. Figure 2 shows an example of the laser wakefield generated in a plasma channel obtained by numerical solution of Eqs. (1a)-(1f). One can see that the wake wave front is curved. This is caused by the radial increase of the unperturbed plasma density in the channel and by the nonlinear increase of the wake wavelength (which has a maximum on the axis) with the amplitude $[5,17,19]$. Another feature of the channel-guided laser wakefield, which can be seen in Fig. 2, is that the amplitude of the
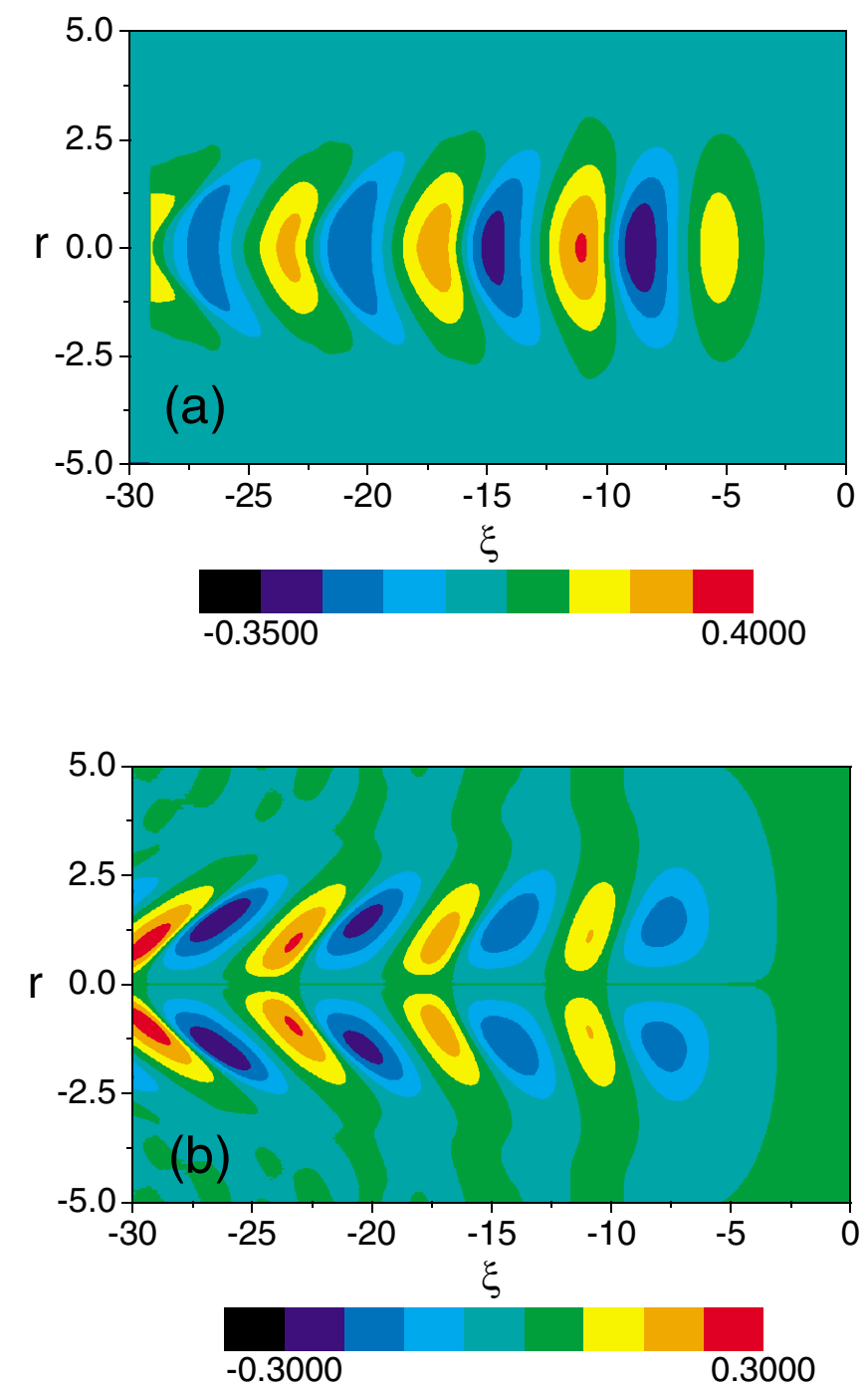

FIG. 2. (Color) The laser wakefield generated in a plasma channel. The normalized longitudinal $E_{z}$ (a) and transverse $f_{r}=\beta_{g} H_{\theta}-E_{r}$ (b) components of the wakefield. $a_{0}=1$, $\sigma_{z}=2, \sigma_{r}=2.5$, and $\xi_{c}=-3 \sigma_{z}$. accelerating (focusing) wakefield component is decreasing (increasing) with increasing distance to the laser pulse [5].

The motion of an electron in the laser wakefield is described by the following equations [15]:

$$
\begin{gathered}
\frac{\partial^{2} \xi}{\partial \tau^{2}}+\frac{1}{\gamma}\left[\left(1-\beta_{z}^{2}\right) E_{z}-\beta_{z} \beta_{r} E_{r}+\beta_{r} H_{\theta}\right]+ \\
\frac{\left(1-\beta_{g} \beta_{z}\right)}{4 \beta_{g}^{2} \gamma^{2}} \frac{\partial a^{2}}{\partial \xi}=0, \\
\frac{\partial^{2} r}{\partial \tau^{2}}+\frac{1}{\gamma}\left[\left(1-\beta_{r}^{2}\right) E_{r}-\beta_{z} \beta_{r} E_{z}-\beta_{z} H_{\theta}\right]- \\
\frac{1}{4 \beta_{g}^{2} \gamma^{2}}\left(\beta_{r} \frac{\partial a^{2}}{\partial \xi}-\frac{1}{\beta_{g}} \frac{\partial a^{2}}{\partial r}\right)=0,
\end{gathered}
$$

where $\gamma=\left[\left(1+a^{2} / 2\right) /\left(1-\beta_{z}^{2}-\beta_{r}^{2}\right)\right]^{1 / 2}=\left(1+p_{z}^{2}+\right.$ $\left.p_{r}^{2}+a^{2} / 2\right)^{1 / 2}$ is the relativistic factor and the normalized components of the electron velocity can be found from $\beta_{z}=\beta_{g}(1+d \xi / d \tau)$ and $\beta_{z}=\beta_{g} d r / d \tau$. Note that Eqs. (5) include both the wakefield and the ponderomotive force of the laser field. Electrons accelerating in the wake wave (behind the laser pulse, where $a=0$ ) gain energy according to the equation $d \gamma / d \tau \approx-E_{z}$ and undergo damped betatron oscillations in the radial direction [15]. An important integral of motion (Hamiltonian), which is used below, follows from the equation of motion (see, e.g., Ref. [15]):

$$
\gamma-\beta_{g} p_{z}-\phi=\text { const. }
$$

\section{B. One-dimensional (1D) theory}

We start with a 1D theory of trapping and compression of an $e$-bunch in the laser wakefield, which neglects the transverse variation of the laser field and is valid for electrons moving along the pulse axis. This case is of interest because also in the 3D case the trapped electrons will become concentrated close to the axis, such that their longitudinal dynamics are well described by a 1D theory. In addition, the 1D theory allows a more detailed description of the problem and helps to clarify the underlying physics. The 1D laser wakefield is described by the equation [5]:

$$
\frac{d^{2} \phi}{d \xi^{2}}=\frac{1}{2}\left(\frac{1+a^{2} / 2}{(1+\phi)^{2}}-1\right)
$$

When deriving (7) it was assumed that $(1+\phi)^{2} /(1+$ $\left.a^{2} / 2\right) \gg \gamma_{g}^{-2} \approx\left(\omega_{p /} \omega_{L}\right)^{2} \ll 1, \quad$ where $\quad \gamma_{g}=(1-$ $\left.\beta_{g}^{2}\right)^{-1 / 2}$ is the relativistic factor associated with the wakefield. From Eq. (7) one can obtain the relation between the electrical field and the potential in the wake wave generated behind the laser pulse $(a=0)$ : 


$$
E_{z}^{2}+\frac{\phi^{2}}{1+\phi}=\text { const }=E_{z, \max }^{2}
$$

where $E_{z, \max }$ is the wake wave amplitude.

Suppose that an electron, which copropagates with the laser pulse, is initially ahead of the pulse and moves with a velocity $\nu_{0}$, less than the group velocity of the pulse $\nu_{g}$, which, in turn, is equal to the wake phase velocity. Because the electron moves slower than the laser pulse, it is overtaken by the pulse and can be trapped at some point $\xi_{t r}$ in the wakefield. At the trapping point (or, in other words, at the reflection point) the electron velocity becomes equal to $\nu_{g}$. For realistic laser pulse intensities, the electron cannot be trapped in the region of the pulse because of the decelerating wakefield (see Fig. 1); only fast electrons with initial relativistic factors $\gamma_{0} \approx \gamma_{g}$ can be trapped in the leading edge of the pulse (where the wakefield is weak, $E_{z} \approx 0$ ) due to the accelerating ponderomotive force $[14,15]$. The Hamiltonian (6) allows us to find a relation between the initial energy of the electron, $\gamma_{0}$, and the wake potential at the trapping point [15]:

$$
\gamma_{0}=\gamma_{g}^{2}\left[S-\beta_{g}\left(S^{2}-\gamma_{g}^{-2}\right)^{1 / 2}\right],
$$

where $S=1 / \gamma_{g}-\phi_{t r}, \phi_{t r}$ is the wake potential at the trapping point, $\xi_{t r}$. One can see that the initial momentum of the trapped electron has a minimum, $\gamma_{\min }$, corresponding to the minimum of the wake potential, $\phi_{\min }<0$. At this trapping point the wake electric field $E_{z}$ is zero. The maximum value, $\gamma_{\max }=\gamma_{g}$, corresponds to the point in the wake where $\phi_{t r}=0$ and $E_{z}=$ $-E_{z, \max }<0$. Figure 3 shows the typical dependence of the initial energy of the electron on the position of the trapping point. The trapping region, shown in Fig. 3 between dashed lines, is less than a quarter of the linear

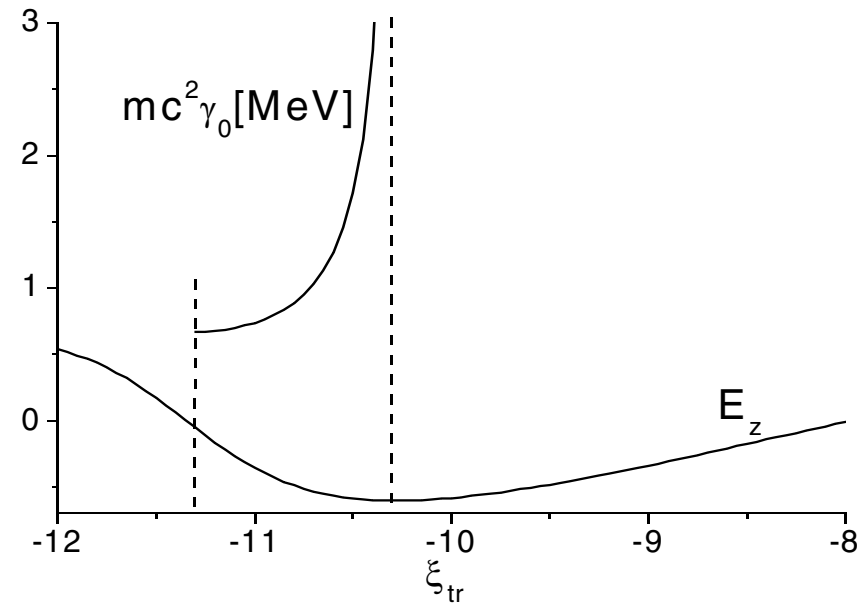

FIG. 3. Initial energy of the trapped electron (in MeVs) and the normalized accelerating field $E_{z}$ in the wake wave in dependence on the trapping point in the wave, $a_{0}=1.5, \gamma_{g}=$ 50 , and $\sigma_{z}=2$. The trapping region is shown between dashed lines. plasma wavelength $\lambda_{p}=2 \pi / k_{p}$. Because of steepening of the plasma wave, the length of the trapping region decreases with increasing wake amplitude $E_{z \text {,max }}$, in spite of the increase of the nonlinear plasma wavelength with increasing $E_{z, \max }$. Thus, any electron with an initial energy $\gamma_{\min }<\gamma_{0}<\gamma_{g}$ will be trapped in this trapping region where the normalized potential is in the range $\phi_{\min }<\phi<0$. The maximum (minimum) wake potential can be expressed through the wake amplitude from (8):

$$
\phi_{\min (\max )}=\mp E_{z, \max }\left(1+E_{z, \max }^{2} / 4\right)^{1 / 2}+E_{z, \max }^{2} / 2 .
$$

In Fig. 3 we see also that all electrons with the initial energy slightly above the minimum value $\gamma_{\min }$ are trapped near the accelerating maximum.

For a linear and moderately nonlinear wakefield with an amplitude much less than the relativistic wavebreaking field, $E_{\text {rel }}=\left[2\left(\gamma_{g}-1\right)\right]^{1 / 2} \quad[5]$, so that $\left(\phi \gamma_{g}\right)^{2} \gg 1$, and supposing that $\gamma_{0}$ is not close to $\gamma_{g}$, one can obtain from (9):

$$
\gamma_{0} \approx\left(\left|\phi_{t r}\right|+1 /\left|\phi_{t r}\right|\right) / 2,
$$

where $-1<\phi_{t r}<0$. Then, for the minimum trapping energy one finds:

$$
\gamma_{\min } \approx\left(\left|\phi_{\min }\right|+1 /\left|\phi_{\min }\right|\right) / 2 .
$$

In the linear wakefield $|\phi| \ll 1$ and therefore $\gamma_{\min } \approx$ $0.5 /\left|\phi_{\min }\right| \gg 1$, which means that only relativistic electrons with $\gamma_{0}>\gamma_{\min }$ can be trapped. However, for an initially relativistic electron bunch, the trapping distance increases in proportion to $\gamma_{0}^{2}$ as calculated below, and can become impractically long. Figure 4 shows numerical results for the minimum trapping energy and the wakefield amplitude in dependence on the peak amplitude $a_{0}$

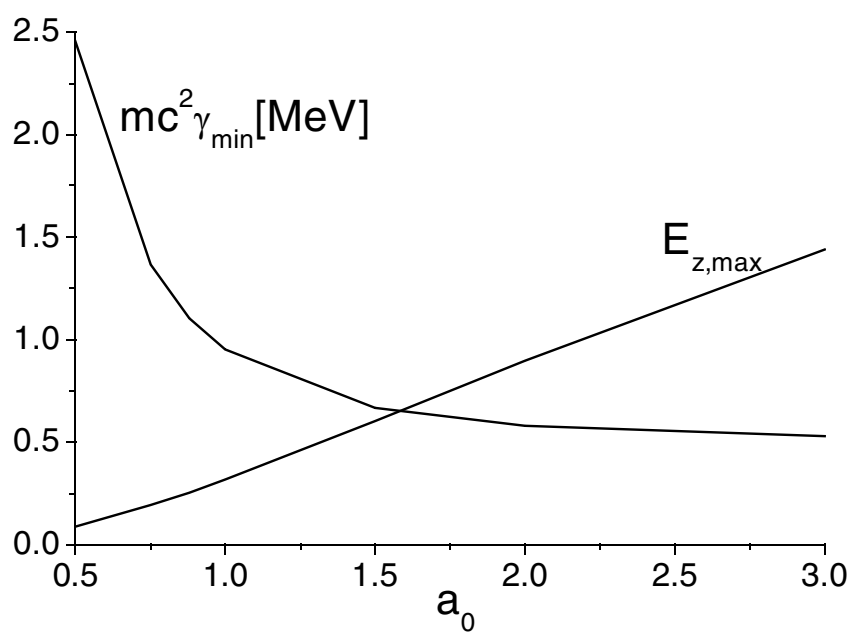

FIG. 4. The minimum trapping energy (in MeVs) and the amplitude of the accelerating field in the wake, $E_{z, \max }$, in dependence on the laser pulse amplitude $a_{0} ; \gamma_{g}=50$ and $\sigma_{z}=2$. 
of the laser pulse. The results confirm the prediction of the analytical calculations that the trapping in a linear wakefield requires initially relativistic electrons.

Let us introduce the trapping time $\tau_{t r}$, which is the time it takes for an electron to reach the trapping point $\xi_{t r}$. Note that according to the normalized variables we use, a time interval is practically equal to a distance the laser pulse propagates during this time interval. Therefore we also introduce the trapping distance, $L_{t r} \approx \tau_{t r}$, which is the distance covered by the pulse during the trapping time. The trapping time depends on the initial energy $\gamma_{0}$ and on the initial position $\xi_{0}$, and can be expressed as

$$
\tau_{t r}\left(\xi_{0}, \gamma_{0}\right)=\tau_{t r 0}\left(\gamma_{0}\right)+\xi_{0} /\left(1-\beta_{0} / \beta_{g}\right)
$$

here $t_{t r 0}$ is the trapping time of an electron which is initially $(\tau=0)$ at a distance $3 \sigma_{z}$ ahead of the pulse center, where the laser field can be considered to be zero. An example of the dependence of the trapping distance $L_{t r}$ on the initial electron energy is shown in Fig. 5 for $a_{0}=2$ and $\gamma_{g}=50$. In this case $E_{z, \max } \approx 0.9$, the nonlinear plasma wavelength is $\Lambda_{p} \approx 1.144 \lambda_{p}$, and $\gamma_{\min } \approx 1.13$. The trapping time has a minimum for some $\gamma_{*}$, which, for the parameters of Fig. 5, corresponds to energy of about $700 \mathrm{keV}$ (kinetic energy of about $200 \mathrm{keV}$ ). Trapping of electrons which have an energy less than or higher than $m_{e} c^{2} \gamma_{*}$ takes longer. For slower particles this is because they are trapped in a wake region with smaller accelerating field and it takes a longer time for these electrons to reach the trapping energy $m_{e} c^{2} \gamma_{g}$. For faster electrons, it takes longer time for the pulse to overtake the electron due to the smaller difference between the laser pulse and the electron velocities. Note, however, that slower electrons actually reach the trapping region in the wake earlier than faster electrons. For the

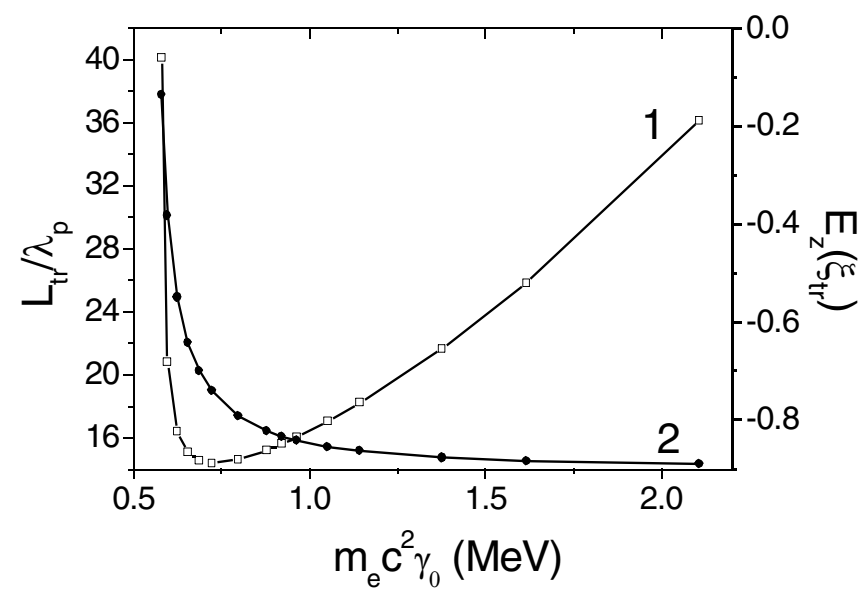

FIG. 5. The trapping distance normalized to the linear plasma wavelength (1) and the strength of the accelerating wakefield at the trapping point (2) depending on the initial energy of an electron; $a_{0}=2, \gamma_{g}=50, \sigma_{z}=2$, and $\xi_{c}=$ $-3 \sigma_{z}$. trapping time of an electron bunch, in which the electron momentum initially changes in the range $\gamma_{1} \leq \gamma_{0} \leq \gamma_{2}$, and, supposing that the tail of the bunch is at $\xi=0$, we can write

$$
\Delta \tau_{t r} \approx \Delta L_{t r} \approx\left(\tau_{t r \text {, max }}-\tau_{t r, \text { min }}\right)+L_{0} /\left(1-\beta_{2} / \beta_{g}\right),
$$

where $\tau_{t r, \max (\min )}$ is the maximum (minimum) value of $\tau_{t r 0}$ for electrons in the range $\gamma_{1} \leq \gamma_{0} \leq \gamma_{2}$, and $L_{0}$ is the dimensionless initial bunch length. When the initial bunch is approximately monoenergetic and $1 \ll \gamma_{0}^{2} \ll$ $\gamma_{g}^{2}$, we have for the bunch's trapping distance:

$$
\Delta L_{t r} \approx 2 \gamma_{0}^{2} L_{0} .
$$

In this case the trapping distance quickly grows with the initial bunch energy.

After the trapped electron has reached a relativistic energy $(\gamma \gg 1)$ in the wakefield, its dynamics in the moving frame becomes slow, and its spatial position in the linear or moderately nonlinear wake changes on a characteristic normalized time scale of $\tau_{d}=2 \pi \gamma_{g}^{2}$. During this time the accelerating electron slips by a distance of $\lambda_{p} / 2$ (that corresponds to a value of $\pi$ in our normalized notation) relative to the wakefield. This time scale is known as the detuning (or dephasing) time [5]. During the dephasing time, the laser pulse propagates over a distance $L_{d} \approx \tau_{d}$, which is typically a few centimeters. Another electron with the same initial energy, but with different initial position $\xi_{0}$, will be trapped very close to the first one if $\Delta \tau_{t r} \ll \tau_{d}$. Then the length of the trapped bunch can be calculated from the expression:

$$
L \approx \Delta L_{t r} / 2 \gamma_{g}^{2} .
$$

In the case described by expression (15) the trapped bunch length is

$$
L \approx\left(\gamma_{0} / \gamma_{g}\right)^{2} L_{0} \ll L_{0} .
$$

According to (17) the trapped bunch length is considerably less than the initial bunch length, so that an extremely short $e$-bunch with $L_{0} \ll \lambda_{p}$ is not needed before acceleration in our injection scheme. Moreover, with our approach one can generate such an extremely short bunch. For example, when $\gamma_{0}=4\left(m_{e} c^{2} \gamma_{0} \approx\right.$ $2 \mathrm{MeV}$ ), $\gamma_{g}=50$, and the initial bunch duration is 500 fs (which corresponds to a bunch length of $150 \mu \mathrm{m}$ ), one finds from (17) a trapped bunch length of only $0.96 \mu \mathrm{m}$ (or just $\approx 3 \mathrm{fs}$ in duration).

Not only the initial bunch length but also the differences in the initial velocities (energies) of bunch electrons contribute to the trapped bunch length because electrons with different $\gamma_{0}$ are trapped at different trapping points (see also Fig. 3). From Eq. (11) it follows that $\phi_{t r} \approx p_{0}-$ $\gamma_{0}$. Accordingly, for an electron bunch with a finite energy 
spread, $\gamma_{1} \leq \gamma_{0} \leq \gamma_{2}$, we can write $\delta \phi_{t r}=\phi_{t r}\left(\gamma_{2}\right)-$ $\phi_{t r}\left(\gamma_{1}\right) \approx\left|E_{z}\right| L \approx \delta \gamma_{0}-\delta p_{0}$, where $E_{z}$ is the wake electric field in the region occupied by the trapped bunch, $L$ is again the trapped bunch length, $\delta p_{0}=p_{2}-p_{1}$, and $\delta \gamma_{0}=\gamma_{2}-\gamma_{1}$. From this, when the effect of the finite initial bunch length is less than the effect of the initial energy spread, the trapped bunch length can be estimated as $L \approx\left(\delta \gamma_{0}-\delta p_{0}\right) /\left|E_{z}\right|$.

Test particle simulations, based on the numerical solutions of Eqs. (1) and (5), indeed showed that the trapped bunch length is much less than the plasma wavelength and undergoes practically no change during acceleration, because all accelerated electrons fly with ultrarelativistic velocities very close to the speed of light. The numerical results are in a good agreement with the above analytical calculations. One can see that the absolute energy spread in the trapped bunch can be estimated from $\delta \gamma \approx$ $\Delta \tau_{t r}\left|E_{z}\left(\xi_{t r}\right)\right|$. In the trapping region $d E_{z} / d \xi<0$, therefore, electrons in the head of the trapped bunch experience a larger accelerating field and gain more energy compared to those in the tail. This increases the absolute energy spread in the bunch. However, the situation changes after the bunch passes the accelerating maximum and slips to the region where $d E_{z} / d \xi>0$. Here the tail (slower) electrons experience a stronger accelerating field, which leads to a decrease in the absolute energy spread. According to our numerical results the relative energy spread, $\varepsilon=\delta \gamma / \gamma$, where $\gamma \approx-\int E_{z} d \tau$, decreases monotonically during acceleration and can reach a value on the order of $1 \%$ [14-16].

Another important issue in laser wakefield acceleration is the so-called beam loading effect. A short $e$-bunch propagating in plasma generates a plasma wakefield [5], which in our case of a short trapped bunch $\left(L \ll \lambda_{p}\right)$, decelerates the bunch electrons. The magnitude of the decelerating field is proportional to the number of electrons in the bunch, so beam loading restricts the number of electrons that can be trapped and accelerated in the wakefield [15]. If the initial bunch energy is close to the minimum energy for trapping, the accelerating field at the trapping point is close to zero (see Fig. 3), so the number of electrons that can be accelerated is quite small. However, with an increase of the initial bunch energy, the accelerating field at the trapping point rapidly attains its maximum value. This allows trapping of more particles before the beam-loading limit is reached, and this limit is calculated below.

\section{Three-dimensional effects}

For a three-dimensional (3D) linear wakefield in a uniform plasma, the transverse force is defocusing in the trapping region, so that in this case electrons cannot be trapped at all. Fortunately, nonlinear wakefields and wakefields generated in a plasma channel $[15,19]$ provide a larger overlap of focusing and accelerating regions, so that our injection scheme is feasible in 3D. Video 1 demonstrates an example of trapping, compression, and acceleration of a low-energy electron bunch $\left(m_{e} c^{2} \gamma_{0} \approx 1.14 \mathrm{MeV}, p_{0}=2\right)$ in a moderately nonlinear laser wakefield $\left(E_{z, \max } \approx 0.88\right.$ in the first accelerating maximum) generated in a plasma channel [20]. In this example the initial bunch is longer than the plasma wavelength, $L_{0} \approx 4.77 \lambda_{p}$ ( $L_{0}=30$ in the normalized units). The last snapshot shows the trapped bunch after the laser pulse has propagated in the plasma channel a distance of $239 \lambda_{p}$. The bunch is trapped near the first accelerating maximum in the wake and is much shorter than the
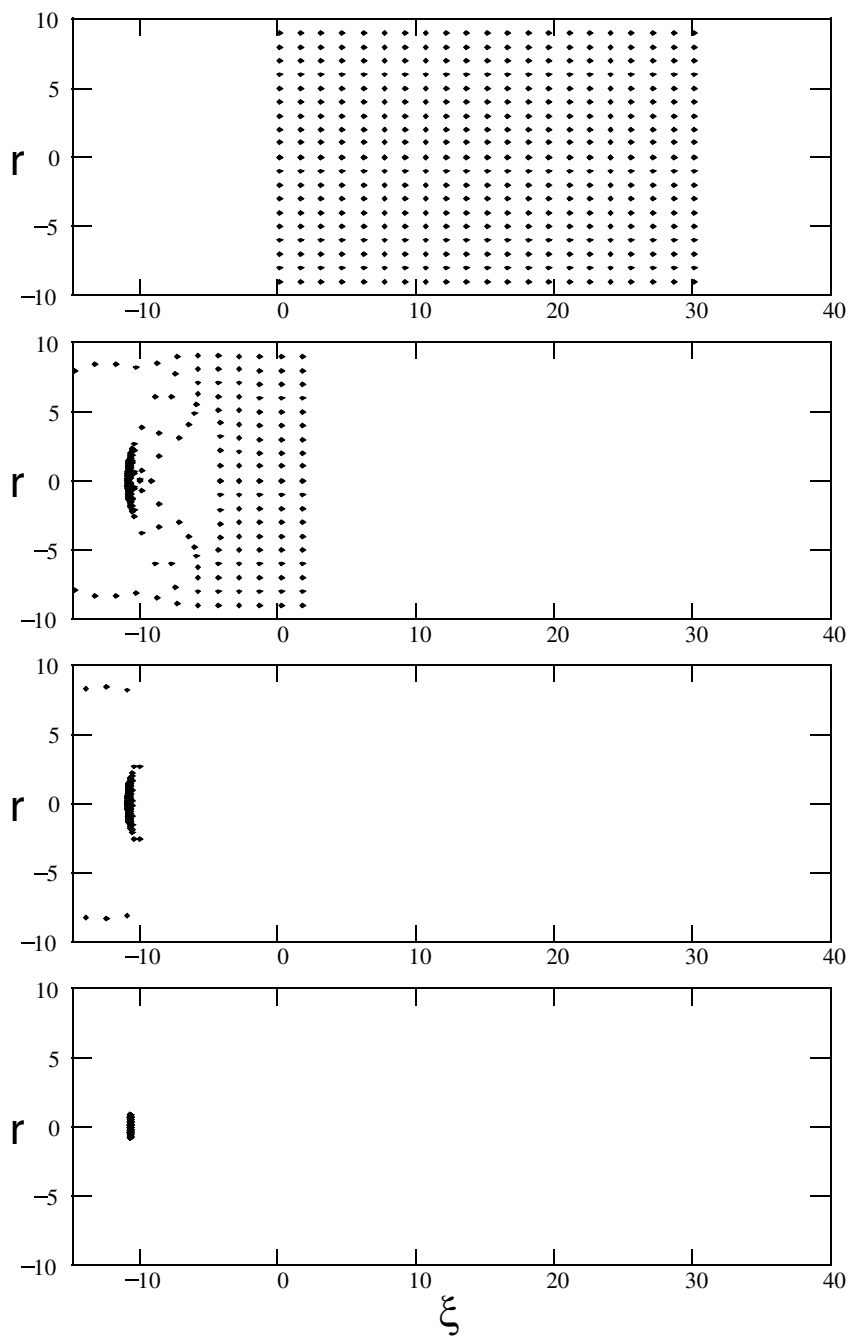

VIDEO 1. The movie and snapshots from it demonstrating the trapping and acceleration of an initially monoenergetic $\left(p_{0}=2, m_{e} c^{2} \gamma_{0} \approx 1.14 \mathrm{MeV}\right) e$-bunch in a channel-guided laser wakefield. The snapshots show the $e$-bunch (dots represent the electrons) at laser pulse propagation distances $0,43 \lambda_{p}$, $62 \lambda_{p}$, and $239 \lambda_{p}$. The initial bunch length is $L_{0} \approx 4.77 \lambda_{p}$, the laser pulse (not shown) parameters are $a_{0}=2, \sigma_{z}=2$, $\sigma_{r}=r_{c h}=5, \gamma_{g}=50$, and the pulse center is located at $\xi_{c}=$ $-3 \sigma_{z}=-6$. 
plasma wavelength, $L \approx \lambda_{p} / 57$; the bunch length is approximately constant during the acceleration. The bunch radius slowly decreases during acceleration (see also Refs. [15,16]) and is $r_{b} \approx 0.14 \lambda_{p}$ in the last snapshot, which gives the accelerated bunch a "pancake" shape, $2 r_{b} \gg L$. The trapped electrons are concentrated near the axis due to the strong focusing force. The off-axis electrons undergo damped betatron oscillations [15]. Note also that electrons, which are initially in the outer periphery $\left(r_{0}>8\right)$, where the wakefield is weak, are not trapped. For obtaining absolute numbers for our example, let us assume a typical plasma wavelength of $\lambda_{p}=$ $40 \mu \mathrm{m}$. In this case the initial bunch length is $L_{0} \approx$ $191 \mu \mathrm{m}$ (the bunch duration of $637 \mathrm{fs}$ ). After the laser pulse has propagated a distance of $239 \lambda_{p} \approx 9.6 \mathrm{~mm}$, the accelerated bunch attains a bunch length of $L \approx 0.7 \mu \mathrm{m}$, a bunch diameter of $2 r_{b} \approx 11 \mu \mathrm{m}$, and an average energy of $585 \mathrm{MeV}$.

When looking at the electron dynamics more closely, one can identify the following. First, when an electron passes the region of the laser pulse it experiences a ponderomotive force from the laser pulse as well as the force from the excited wakefield. The electron will interact first with the rising part of the laser pulse where the ponderomotive force is accelerating and the wakefield is decelerating. It turns out that these forces nearly compensate each other. During the falling part of the laser pulse the ponderomotive force and the wakefield are both decelerating. Because the electron is decelerated in this region, the difference between the laser pulse velocity $\nu_{g}$ and the electron velocity becomes larger and the electron approaches the trapping point faster. This effect is stronger for the near-axis electrons and can be seen in the second snapshot in Video 1-the electrons are "sucked" in the accelerating region. Since the effect of retardation is weaker for the off-axis electrons and because these electrons travel a longer distance to the trapping point, they are trapped in the wakefield later. This leads to a longer trapped bunch as compared to the 1D case, but still the bunch length is much less than the plasma wavelength if the trapping distance is much less than the dephasing length.

In 3D, the trapping conditions cannot be calculated analytically from a single equation (6), as in $1 \mathrm{D}$, because now we have additional parameters-namely initial transverse momentum $p_{r 0}$ and transverse position $r_{0}$ of the electron. Numerical results for the minimum trapping energy $m_{e} c^{2} \gamma_{\min }$ as a function of the initial transverse position are presented in Fig. 6. One can see that the minimum trapping energy has a minimum on axis, grows with $r_{0}$, and reaches relativistic values for electrons in the periphery of the laser pulse spot, $r_{0}>(1-2) \sigma_{r}$. Therefore, in 3D one should use an initial $e$-bunch with sufficiently high energy to provide trapping of off-axis electrons. Since the trapped bunch sizes are much less

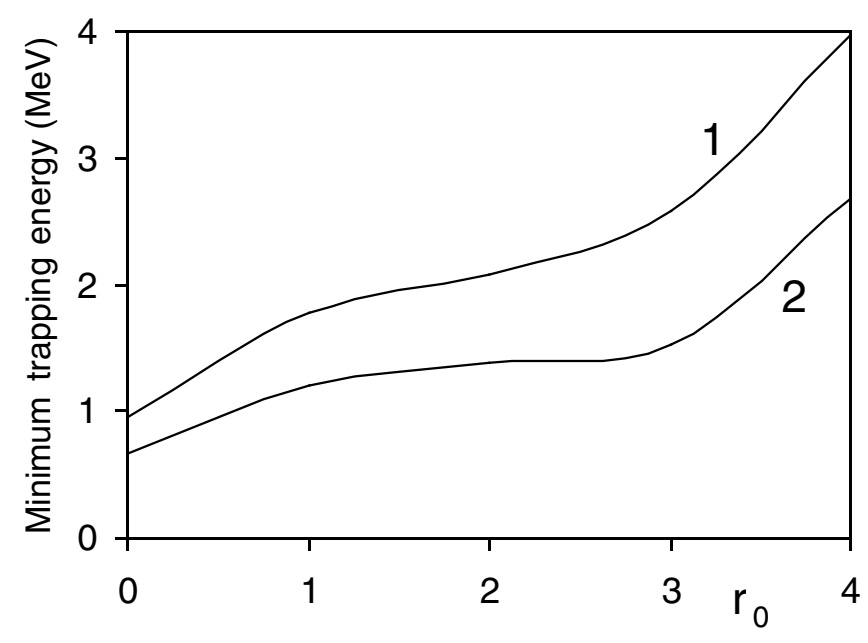

FIG. 6. The minimum trapping energy (in MeVs) depending on the initial normalized radial position $r_{0}$. The initial transverse momentum is zero, $\sigma_{z}=2$ and $\sigma_{r}=r_{c h}=2.5$. (1): $a_{0}=1$ (peak intensity of $I_{0} \approx 2.11 \times 10^{18} \mathrm{~W} / \mathrm{cm}^{2}$ for a laser wavelength of $\left.\lambda_{L}=0.8 \mu \mathrm{m}\right) ;(2): a_{0}=1.5\left(I_{0} \approx 4.75 \times\right.$ $10^{18} \mathrm{~W} / \mathrm{cm}^{2}$ ).

than the characteristic spatial extent of the accelerating region, the electrons experience approximately the same accelerating field. The latter, in turn, can provide a small energy spread in the accelerating bunch. Because the trapped electrons remain close to the axis during acceleration, their longitudinal dynamics are well described by the 1D theory [15].

Let us estimate the beam-loading limit in 3D. Suppose that $r_{\max }=b \sigma_{r}$ is the maximum initial distance from the axis for the bunch electrons, which can be trapped in the laser wakefield (the value of the parameter $b$ depends on the laser pulse and initial bunch parameters), and $L_{0}$ is the initial bunch length. Then, the total number of trapped electrons can be calculated from the expression:

$$
N_{\text {tot }}=\pi n_{b 0} b^{2} \sigma_{r}^{2} L_{0} / k_{p}^{3},
$$

where $n_{b 0}$ is the electron concentration in the initial bunch and $\sigma_{r}$ and $L_{0}$ are normalized. The maximum normalized decelerating field (which is generated by the bunch itself) is $E_{b, \text { max }} \approx T L n_{b} / n_{p}$ [21,22], here $n_{b}=N_{\text {tot }} k_{p}^{3} /\left(\pi R^{2} L\right)$ is the electron concentration in the trapped bunch, $L \ll 1$ and $R$ are the dimensionless length and radius of the bunch, and $T=1-R K_{1}(R)<1, K_{1}(R)$ being the modified Bessel function. The beam-loading restriction implies that $E_{b, \text { max }} \ll E_{z, \text { max }}, E_{z \text {, max }}$ is the laser wake wave amplitude. This leads to the following restriction on the total number of electrons in the accelerating bunch:

$$
N_{\text {tot }} \ll \frac{\pi R^{2} n_{p} E_{z, \max }}{T k_{p}^{3}} \approx 1.4 \times 10^{7}\left(R^{2} / T\right) E_{z, \max } \lambda_{p}[\mu \mathrm{m}] .
$$

For example, when $E_{z, \max }=0.6\left(a_{0} \approx 1.5\right), \lambda_{p}=40 \mu \mathrm{m}$ 
$\left(n_{p} \approx 7 \times 10^{17} \mathrm{~cm}^{-3}\right)$, and $R=0.5$ one has $N_{\text {tot }} \ll 5 \times$ $10^{8}$. Note that expression (19) does not contain the trapped bunch length. From (18) and (19) one can also find restrictions on the initial parameters of the bunch.

The plasma wakefield generated by the bunch is selffocusing. The maximum self-focusing force for the trapped bunch $(L \ll 1, R<1)$ can be estimated from the expression $\left(f_{r, b}\right)_{\max } \approx L^{2} R K_{1}(R) n_{b} / 4 n_{p}$ [22], $R K_{1}(R)<1$. The maximum focusing force acting on the bunch in the laser wakefield can be calculated as $\left(f_{r}\right)_{\max } \approx\left|\partial f_{r} / \partial r\right| R \approx 2 R E_{z, \max } / \sigma_{r}$, where we assumed that $\left|f_{r}\right|$ reaches its maximum at $\sigma_{r} / 2$ and that the maximum focusing and accelerating fields in the laser wakefield are comparable (see Fig. 2). For typical parameters of the problem, the laser wakefield focuses the trapped bunch much stronger than the self-focusing field of the bunch.

\section{LASER PULSE DYNAMICS}

The energy of a laser pulse with the Gaussian profile (3) is $W_{L}=(\pi / 2)^{3 / 2} \sigma_{z} \sigma_{r}^{2} I_{0} / c$, here $\sigma_{z, r}$ are not normalized and $I_{0}=m_{e}^{2} c^{3} \omega_{L}^{2} a_{0}^{2} / 8 \pi e^{2}$ is the peak intensity, or, in practical units:

$$
W_{L}[\mathrm{~J}] \approx 9 \times 10^{-5} \frac{\sigma_{z}[\mu \mathrm{m}] \sigma_{r}^{2}[\mu \mathrm{m}]}{\lambda_{L}^{2}[\mu \mathrm{m}]} a_{0}^{2},
$$

where the laser wavelength $\lambda_{L}$ and $\sigma_{z, r}$ are in microns. The main mechanism of energy loss for a short laser pulse in a fully ionized plasma channel is the generation of the laser wakefield. Let us calculate the energy transferred to the laser wakefield supposing that the wakefield parameters change insignificantly during propagation of the laser pulse in a plasma channel. The wakefield energy can be represented as the sum of the electromagnetic (EM) field energy and the kinetic energy of plasma electrons, which, in average, is equal to the EM field energy. The density of the EM energy is $\left(\mathbf{E}^{2}+\mathbf{H}^{2}\right) / 8 \pi$. Then, averaging over the wake wave oscillations, for the energy transferred to the laser wakefield we obtain: $W_{\text {wake }}=\left(l_{\text {prop }} / 4\right) \int\left(E_{z 0}^{2}+\right.$ $\left.E_{r 0}^{2}+H_{\theta 0}^{2}\right) r d r$, where $l_{\text {prop }}$ is the laser pulse propagation distance in the plasma, $E_{z 0, r 0}(r)$ and $H_{\theta 0}(r)$ are the amplitudes of the corresponding wakefield components, which are dependent on $r$. Taking into account that $E_{z 0} \propto$ $a^{2}$ one obtains $E_{z 0} \approx E_{z, \max } \exp \left(-2 r^{2} / \sigma_{r}^{2}\right)$. Next, according to (1e) $E_{r 0}, H_{\theta 0} \propto d E_{z 0} / d r \propto r \exp \left(-2 r^{2} / \sigma_{r}^{2}\right)$ and we can write $E_{r 0}=E_{r, \max } y(r), H_{\theta 0}=H_{\theta, \max } y(r)$, where $E_{r, \text { max }}$ and $H_{\theta, \text { max }}$ are the maximum values of the amplitudes $E_{r 0}(r)$ and $H_{\theta 0}(r)$, which are reached at $r=\sigma_{r} / 2$, and $y(r)=\left(2 e^{1 / 2} r / \sigma_{r}\right) \exp \left(-2 r^{2} / \sigma_{r}^{2}\right), e \approx 2.72$ is the base of the natural logarithm. Finally, for the wake wave energy we find:

$$
\begin{aligned}
W_{\text {wake }} & \approx l_{\text {prop }} \sigma_{r}^{2} E_{z, \text { max }}^{2}(1+h), \\
h & =e\left(E_{r, \text { max }}^{2}+H_{\theta, \text { max }}^{2}\right) / E_{z, \text { max }}^{2} .
\end{aligned}
$$

According to our numerical calculations for a moderately nonlinear wakefield $\left(E_{z, \max }<1\right) E_{z, \max } \approx$ $0.4 E_{W B} a_{0}^{2} /\left(1+a_{0}^{2} / 2\right)^{1 / 2}$ (see also Ref. [5]) when $\sigma_{z}=$ $\lambda_{p} / \pi$ (optimal condition for the laser wakefield generation). Taking into account these relations we can find the ratio of the wake wave energy to the initial laser pulse energy:

$$
\varepsilon_{W} \approx 0.2(1+h) \frac{a_{0}^{2}}{1+a_{0}^{2} / 2}\left(\frac{\lambda_{L}}{\lambda_{p}}\right)^{2} \frac{l_{\text {prop }}}{\lambda_{p}} .
$$

Note that in (22) $\left(l_{\text {prop }} / \lambda_{p}\right)\left(\lambda_{L} / \lambda_{p}\right)^{2}=l_{\text {prop }} / l_{d}$, where $l_{d}=\lambda_{p}^{3} / \lambda_{L}^{2}$ is the dimensional dephasing length. The relative energy change of the laser pulse during the $e$-bunch trapping can be estimated from (22) by substituting the propagation distance by the trapping distance. When $\varepsilon_{w} \ll 1$ one can expect an insignificant change in the laser pulse energy during propagation in the plasma channel. For example, when $\lambda_{p}=40 \mu \mathrm{m} \quad\left(n_{p}=\right.$ $\left.7 \times 10^{17} \mathrm{~cm}^{-3}\right), \quad \lambda_{L}=0.8 \mu \mathrm{m}, \quad l_{\text {prop }}=4 \mathrm{~cm}, \quad a_{0}=1$, and, supposing that $h=1$ (which is approximately the case for realistic laser pulse parameters), one finds $\varepsilon_{w} \approx$ 0.1 . Expression (22) allows to estimate the energy depletion distance $l_{\text {dep }}$, i.e., the distance on which the laser pulse loses half of its energy: $l_{\mathrm{dep}} / l_{d} \approx 2.5(1+$ $\left.a_{0}^{2} / 2\right) /\left[a_{0}^{2}(1+h)\right]$. One can see that for high-intensity laser pulses, which are required for our LWFA injection scheme, the energy depletion distance can be on the order of the dephasing length or longer, so that the laser pulse energy depletion does not restrict the applicability of the scheme.

If looked upon to further detail, the dynamics of a short intense laser pulse in a plasma channel can be quite complicated (see, e.g., Ref. [23] and references therein). In the region occupied by the laser pulse the plasma parameters are changed because of the generated wakefield. This locally changes the plasma dispersion properties and leads to different dynamics for different parts of the pulse. The effect is weak at the pulse head and becomes stronger to the pulse tail, which leads to asymmetric pulse deformation [23]. Because the total number of photons in the pulse, during propagation in fully ionized plasma, is conserved [24] and the pulse loses energy due to the wake wave generation, the frequency of the photons decreases. The effect of frequency decrease is weak at the pulse head and becomes stronger approaching the pulse tail [23]. The decrease in frequency results in an increase of the pulse amplitude (recall that $a \propto 1 / \omega_{L}$ ). The group velocity dispersion leads to compression of the pulse and also contributes to an increase in amplitude [23]. Because of this, the peak power, $P[\mathrm{GW}] \approx 21.5\left(a_{0} \sigma_{r} / \lambda_{L}\right)^{2}$, can become comparable to the critical power for the selffocusing, $P_{c}[\mathrm{GW}] \approx 17\left(\lambda_{p} / \lambda_{L}\right)^{2}$ [18]. In this case the pulse will be focused, which, in turn, leads to further increase of the peak amplitude $a_{0}$. However, according to 


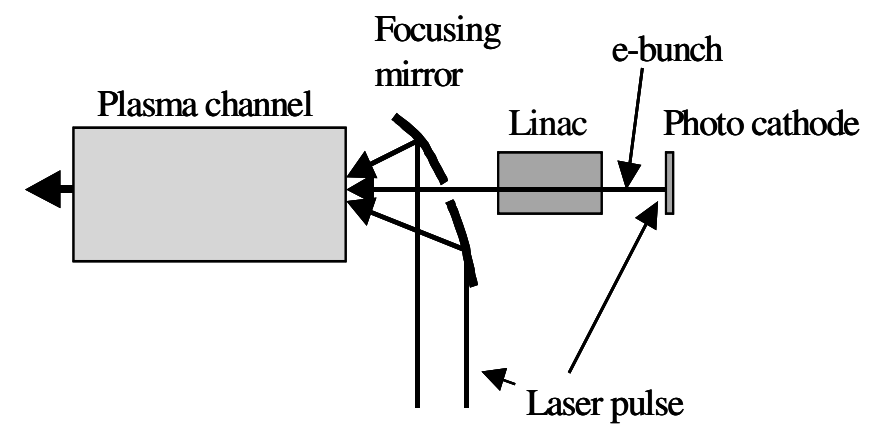

FIG. 7. A possible experimental setup for demonstration of extremely short $e$-bunch generation.

(22), for higher amplitude the pulse energy loss is increased. Because of that the effect of energy loss dominates at some moment and the peak intensity drops relatively fast. Nevertheless, when initially the peak power is less than the critical power and the laser spot size is matched to the plasma channel radius, the energy depletion length $l_{\text {dep }}$ calculated above provides a reasonable scale of the change in the parameters of the pulse. If the trapping distance in our LWFA scheme is much less than the energy depletion length, one can assume that the laser pulse parameters are constant during the trapping.

\section{POSSIBLE EXPERIMENTAL SETUP}

A schematic drawing of a possible experimental setup for demonstration of the extremely short $e$-bunch generation is presented in Fig. 7. A small part of a multiterawatt femtosecond laser pulse is converted to a fs UV pulse and sent to the photocathode. The use of a metal (e.g., $\mathrm{Cu}$ or $\mathrm{Mg}$ ) photocathode is preferable for generation of subpicosecond $e$-bunches. Electrons emitted by the photocathode are accelerated by the rf field in a linac to a few MeVs and focused to a plasma channel through a small hole in a parabolic focusing mirror; as we have mentioned in the Introduction, subpicosecond $e$-bunches can be generated by current technology. The major part of the pulse is directed to the focusing mirror that focuses it to a high intensity (on the order of $10^{18} \mathrm{~W} / \mathrm{cm}^{2}$ ). The focused laser pulse enters the plasma channel just after the low-energy $e$-bunch from the rf linac and generates the laser wakefield, which can trap and accelerate the electrons.

Creation of a parabolic plasma channel for efficient guiding of a high-intensity laser pulse has been demonstrated in experiments [18]. The time jitter between the initial $e$-bunch and the laser pulse, which drives the laser wakefield, on the order of a picosecond is acceptable; in this case the trapping distance is still much less than the plasma channel length and the accelerated bunch parameters change insignificantly. Because the initial $e$-bunch and the laser pulse focused to the plasma channel originate from the same laser pulse, the time delay between the bunch and the laser wakefield can be controlled with an optical delay line to an accuracy of much less than $1 \mathrm{ps}$. On the other hand, the time jitter between the rf field and the laser pulse, which drives the photoinjector can be made as small as a few tens of femtoseconds [25]. Thus, the time jitter can be made much less than 1 ps. Note that the initial $e$-bunch should also be focused (by a magnetic field) before it enters the plasma channel. The energy and the energy spread in the bunch accelerated by the laser wakefield can be analyzed by the use of a bending magnet. The energy spread can provide also information about the accelerated bunch length.

In Table I we summarize the calculated parameters for the laser pulse, plasma, and $e$-bunch in the case of $\sim 1 \mathrm{~J}$ laser pulse and the pulse full-width-at-half-maximum duration of (30-50) fs for intensity. The parameters for the laser pulse, plasma, and $e$-bunch are within the pos-

TABLE I. Parameters for proof-of-principle experiment with $\sim 1 \mathrm{~J},(30-50)$ fs laser pulse.

Laser pulse

Plasma channel

Injected electron bunch

Accelerated electron bunch
Wavelength: $0.8 \mu \mathrm{m}$

Peak intensity at focus : $(1-6) \times 10^{18} \mathrm{~W} / \mathrm{cm}^{2}$

Normalized amplitude, $a_{0}: 0.7-1.7$

$\sigma_{z}: 7.65-12.75 \mu \mathrm{m}$

$$
\sigma_{r}: 20-50 \mu \mathrm{m}
$$

On-axis electron concentration, $n_{p}(0):(0.7-2) \times 10^{18} \mathrm{~cm}^{-3}$

On-axis plasma wavelength, $\lambda_{p}:(24-40) \mu \mathrm{m}$

Channel length: $(2-5) \mathrm{cm}$

Energy $m_{e} c^{2} \gamma_{0}:(1-4) \mathrm{MeV}$

Bunch duration: (200-700) fs

Bunch diameter: (100-200) $\mu \mathrm{m}$

Number of electrons: $10^{8}-10^{9}[(16-160) \mathrm{pC}]$

Energy $m_{e} c^{2} \gamma_{0}:(0.2-4.5) \mathrm{GeV}$

Bunch duration: (1-10) fs

Bunch diameter: $(2-10) \mu \mathrm{m}$

Number of electrons: up to $10^{8}(16 \mathrm{pC})$ - beam-loading limit 
sibilities of current technologies. At the initial trapping stage the laser pulse can be focused to the plasma channel to a spot size less than the matched value for guiding to provide sufficient high intensity to trap slower electrons for which the trapping distance is shorter. In this case the trapped bunch will be accelerated further in the plasma channel at lower laser intensity.

\section{CONCLUSION}

Our results show the possibility of generating relativistic electron bunches with a few femtoseconds duration in a laser wakefield accelerator scheme when the initial $e$-bunch is injected in front of the laser pulse. Such an extremely short bunch is required in many applications and cannot be produced by conventional methods. Our approach does not require preparing and injecting in the wakefield an extremely short electron bunch, and it allows the achievement of controlled laser wakefield acceleration with a good quality of the accelerated bunch. Current technology can provide all three components needed in our LWFA scheme: a high-intensity laser pulse, a plasma channel, and a low-energy, subpicosecond electron bunch.

Recently, after submission of this paper, new promising experimental results were published in Nature (London) [26-28]. In these experiments $e$-bunches of about $10 \mathrm{fs}$ in duration, with energies around $100 \mathrm{MeV}$ and an energy spread of $2 \%-24 \%$ were generated when a multiterawatt laser pulse interacted with a relatively high density, $\sim 10^{19} \mathrm{~cm}^{-3}$, plasma. In this regime, which can be reached in a narrow range of parameters [28], some plasma electrons are trapped and accelerated in the wakefield due to transverse wave breaking after the nonlinear evolution of the laser pulse in the plasma. The results show a considerable improvement in the $e$-bunch quality compared to previous results obtained in the self-modulated regime [5]. However, the experiments also show a poor shot-to-shot stability $[29,30]$. Because of the high plasma density in this regime, the dephasing length is on the order of a millimeter that limits the energy of a goodquality accelerated bunch. Therefore it seems to be not easy to generate a good-quality femtosecond $e$-bunch in the $\mathrm{GeV}$ range in this regime. The approach we describe in this paper is more flexible, it works in a relatively broad range of parameters and can provide the generation of a few femtosecond $e$-bunches with energies up to a few GeVs.

\section{ACKNOWLEDGMENTS}

This work has been supported by the Dutch Foundation for Fundamental Research on Matter (FOM) under the "Laser Wakefield Accelerators" programme and by the Research Councils U.K. under the Alpha-X laser wakefield acceleration programme [31].
[1] X. J. Wang, X. Qiu, and I. Ben-Zvi, Phys. Rev. E 54, R3121 (1996); M. Uesaka et al., IEEE Trans. Plasma Sci. 28, 1133 (2000).

[2] P. Kung, H.-C. Lihn, H. Wiedemann, and D. Bocek, Phys. Rev. Lett. 73, 967 (1994).

[3] B. E. Carlsten and S. J. Russell, Phys. Rev. E 53, R2072 (1996).

[4] K. J. Kim, S. Chattopadhyay, and C.V. Shank, Nucl. Instrum. Methods Phys. Res., Sect. A 341, 351 (1994).

[5] E. Esarey, P. Sprangle, J. Krall, and A. Ting, IEEE Trans. Plasma Sci. 24, 252 (1996).

[6] H. Kotaki et al., Phys. Plasmas 9, 1392 (2002).

[7] S.V. Bulanov, F. Pegoraro, A. M. Pukhov, and A.S. Sakharov, Phys. Rev. Lett. 78, 4205 (1997).

[8] S. Bulanov, N. Naumova, F. Pegoraro, and J. Sakai, Phys. Rev. E 58, R5257 (1998).

[9] P. Tomassini et al., Phys. Rev. ST Accel. Beams 6, 121301 (2003).

[10] D. Umstadter, J. K. Kim, and E. Dodd, Phys. Rev. Lett. 76, 2073 (1996).

[11] E. Esarey, R. F. Hubbard, W. P. Leemans, A. Ting, and P. Sprangle, Phys. Rev. Lett. 79, 2682 (1997).

[12] H. Kotaki, S. Masuda, M. Kondo, J. K. Koga, and K. Nakajima, Phys. Plasmas 11, 3296 (2004).

[13] See also recent theoretical and experimental results in 11th Advanced Accelerator Concepts Workshop, AIP Conf. Proc. No. 737 (AIP, New York, 2004).

[14] A. G. Khachatryan, JETP Lett. 74, 371 (2001).

[15] A. G. Khachatryan, Phys. Rev. E 65, 046504 (2002).

[16] A. G. Khachatryan, F. A. van Goor, and K.-J. Boller, in Proceedings of the Particle Accelerator Conference (PAC'03), Portland, OR, 2003, edited by J. Chew, P. Lucas, and S. Webber (IEEE, Piscataway, NJ, 2003), pp. 1900-1902.

[17] A. G. Khachatryan, Phys. Rev. E 60, 6210 (1999).

[18] E. Esarey, P. Sprangle, J. Krall, and A. Ting, IEEE J. Quantum Electron. 33, 1879 (1997).

[19] N.E. Andreev, L. M. Gorbunov, V. I. Kirsanov, K. Nakajima, and A. Ogata, Phys. Plasmas 4, 1145 (1997).

[20] See also our website: http://www.tn.utwente.nl/lf/

[21] R. Keinigs and M. Jones, Phys. Fluids 30, 252 (1987).

[22] A. G. Khachatryan, A.Ts. Amatuni, E.V. Sekhposyan, and S. S. Elbakian, Fiz. Plazmy 22, 638 (1996) [Plasma Phys. Rep. 22, 576 (1996)].

[23] D. F. Gordon et al., Phys. Rev. Lett. 90, 215001 (2003).

[24] S.V. Bulanov, I. N. Inovenkov, V. I. Kirsanov, N. M. Naumova, and A. S. Sakharov, Phys. Fluids B 4, 1935 (1992).

[25] F. B. Kiewiet, A. H. Kemper, O. J. Luiten, G. J. H. Brussaard, and M. J. van der Wiel, Nucl. Instrum. Methods Phys. Res., Sect. A 484, 619 (2002).

[26] S.P. D. Mangles et al., Nature (London) 431, 535 (2004).

[27] C.G.R. Geddes et al., Nature (London) 431, 538 (2004).

[28] J. Faure et al., Nature (London) 431, 541 (2004).

[29] T. Katsouleas, Nature (London) 431, 515 (2004).

[30] J. Faure (private communication).

[31] http://phys.strath.ac.uk/alpha-x/ 